Kansas State University Libraries

New Prairie Press

\title{
SPLINE MODELS FOR ESTIMATING HEAT STRESS THRESHOLDS IN CATTLE
}
A. M. Parkhurst
D. A. Spiers
G. L. Hahn

Follow this and additional works at: https://newprairiepress.org/agstatconference

Part of the Agriculture Commons, and the Applied Statistics Commons

\section{(c) (1) $\Theta(9$}

This work is licensed under a Creative Commons Attribution-Noncommercial-No Derivative Works 4.0 License.

\section{Recommended Citation}

Parkhurst, A. M.; Spiers, D. A.; and Hahn, G. L. (2002). "SPLINE MODELS FOR ESTIMATING HEAT STRESS THRESHOLDS IN CATTLE," Conference on Applied Statistics in Agriculture. https://doi.org/10.4148/ 2475-7772.1206

This is brought to you for free and open access by the Conferences at New Prairie Press. It has been accepted for inclusion in Conference on Applied Statistics in Agriculture by an authorized administrator of New Prairie Press. For more information, please contact cads@k-state.edu. 


\title{
SPLINE MODELS FOR ESTIMATING HEAT STRESS THRESHOLDS IN CATTLE
}

\author{
A. M. Parkhurst ${ }^{1}$, D. A.Spiers ${ }^{2}$, T.L.Mader ${ }^{1}$, and G. L. Hahn ${ }^{3}$ \\ ${ }^{1}$ University of Nebraska - Lincoln \\ ${ }^{2}$ University of Missouri - Columbia \\ ${ }^{3}$ U.S. Meat Animal Research Center, U.S. Department of Agriculture
}

\begin{abstract}
Studies of the relationship between animal body temperature and air temperature suggest body temperature is essentially unresponsive until a threshold is reached, then it responds dramatically to increasing air temperature. The goal is to estimate the threshold between the thermoneutral plateau and the beginning of the heat stress challenge. One approach is to fit a polynomial to estimate the knot position and use spline functions to perform linear least squares piecewise polynomial fitting. Another alternative is to use nonlinear regression to estimate the knot or an inflection point of a nonlinear function. In both approaches the cyclic nature of body temperature is ignored. This paper explores the use of nonlinear regression to estimate the knot position and handles the hysteresis effect resulting from the cyclic nature of body temperature. Models are fit to data collected from cattle in chambers subjected to semicontrolled sinusoidal air temperature at the University of Missouri-Columbia Animal Science department and a procedure for estimating the heat stress threshold is proposed.
\end{abstract}

KEY WORDS: Hysteresis, nonlinear regression, nonlinear mixed models, heat stress, thresholds

\section{Introduction}

A heat-stress threshold is the point at which a thermally challenged animal activates a physiological process (e.g., dynamic changes in body temperature) to dissipate the increasing heat load. It is important to identify a threshold for the onset of heat stress since it represents an animal's heat tolerance. This tolerance has a direct impact on productive performance responses such as growth rate, feed efficiency, reproduction, and in extreme environments, survival. Estimates of the heat-stress thresholds may be used generally as a basis for initiating management practices or individually to rank animals according to their heat tolerance.

The relationship between animal body temperature, $\mathrm{Tb}$, and air temperature, $\mathrm{Ta}$, is frequently a topic of investigation (e.g., Mader et. al., 2001). Typically body temperature responds only minimally to air temperature within the thermoneutral range, but rises sharply following the onset of a heat challenge. Several methods are used to estimate the heat-stress threshold. They range from visual examination of a change in the data plots to fitting a statistical model. Often the model is a polynomial, or in the case of spline functions, a piecewise polynomial. The threshold may be the abscissa, Ta, at the optimal value of the polynomial or, when spline functions are used, the threshold is the knot, or the value of Ta at the join point of the polynomials. Frequently, the thresholds (knots) are assumed known and linear regression is used to fit the model. Less frequently, the threshold is considered unknown. When unknown, the threshold can be estimated using the iterative procedures in nonlinear regression. 
Other issues worthy of consideration are animal variation and hysteresis. Rarely are animals homogeneous with respect to body temperature. Variation among individual steers can obscure evocative patterns (Figure 1). Examination of animal variation can lead to valuable biological insights, as well as, more efficient statistical analyses. The same can be said for the issue of hysteresis. By identifying the time sequence of observations for a given animal, the lag or delay between $\mathrm{Tb}$ and $\mathrm{Ta}$ becomes apparent. The duality in $\mathrm{Tb}$ for a given $\mathrm{Ta}$ becomes an important consideration. (See section 3 below). In this paper we discuss how the estimate of heat threshold changes depending on the model selected, as well as, assumptions made about hysteresis, and assumptions about animal variation. To illustrate the models, data from one animal (a crossbred steer) exposed to controlled sinusoidal heat stress at the U.S. Meat Animal Research Center under the direction of G.L.Hahn was used. The full analysis of the motivating experiment contained data from six steers exposed to semi-controlled sinusoidal conditions at the University of Missouri-Columbia Animal Science department under the direction of D.A. Spiers.

\section{Models Considered}

The types of models considered were a) polynomial, b) spline with knot given, and c) spline with knot unknown (Figure 2).

\section{2a.) Polynomial Model}

The only polynomial considered for linear least squares analysis was a quadratic form. The threshold was estimated as the $\mathrm{Ta}$ for which the rate of change in $\mathrm{Tb}$ is zero.

$$
\begin{gathered}
T_{b}=a+b T_{a}+c T_{a}^{2} \\
\frac{\partial T_{b}}{\partial T_{a}}=b+2 c T_{a}=0 \\
T_{a}=-\frac{b}{2 c}
\end{gathered}
$$

Figure 3 is an example of a quadratic estimate for a single steer.

\section{2b.) Spline Model with Knot Given}

Splines are defined here to be piecewise polynomials of degree n (Smith, 1979). The points where the pieces (segments) meet are called join points. The values of the abscissa at the joint points are called knots. It is easier to fit a spline with known knots because linear least squares or maximum likelihood regression methods can be applied. Models can be evaluated using rsquared, mean square error (mse) and and information criteria such as Akaike's AIC and Schwartz's Bayeasan, BIC. The number of segments and values for the knots may be found in the literature, given by theory, estimated by polynomials or obtained from visual examination of data graphs.

Examination of the heat stress data indicates the relationship between $\mathrm{Tb}$ and $\mathrm{Ta}$ for a single steer appears to have two segments (Figure 3). The Tb seems unaffected by Ta until the threshold is reached, then increases noticeably. Thus, we began by fitting a spline of two straight lines with known knot. The value of the knot is judiciously taken from the literature to be $21.5 \mathrm{C}$ (e.g. Hahn, 1980). 
The spline model can be represented in terms of the abscissa, $\mathrm{X}$ for the first segment of Ta, and an additional variable on the abscissa, XII, to provide a continuous scale for the second segment of Ta (Freund and Littell, 2000).

$$
\mathrm{XII}=\max (\mathrm{X}-\mathrm{knot}, 0)
$$

$$
\begin{aligned}
& \text { Model } \mathbf{Y = a}+\mathbf{b}^{*} \mathbf{X}+\mathbf{b I I} * \mathbf{X I I} \\
& \begin{aligned}
\text { If } \mathbf{X}<\mathbf{k n o t} \text { then } \mathbf{Y}=\mathbf{a}+\mathbf{b}^{*} \mathbf{X} \\
\text { If } \mathbf{X}>\mathbf{k n o t} \text { then } \mathbf{Y}=\mathbf{a}+\mathbf{b}^{*} \mathbf{X}+\mathbf{b I I} * \mathbf{X I I} \\
=\mathbf{a}+\mathbf{b} * \mathbf{X}+\mathbf{b I I} *(\mathbf{X}-\mathbf{K N O T}) \\
\mathbf{Y}=(\mathbf{a}-\mathbf{b I I} * \mathbf{K N O T})+(\mathbf{b}+\mathbf{b I I}) * \mathbf{X}
\end{aligned}
\end{aligned}
$$

\section{When $\mathbf{X}=$ Knot both equations give same $\mathbf{Y}$}

This spline model is continuous at the knot (threshold). The above model is a weighted sum of a single constant, a line for the first segment and a line for the second segment. If the coefficient, $\mathrm{b}$, is restricted to zero, the line for the first segment is a plateau. The SAS procedures proc mixed and proc reg can be used for the analysis (SAS, 1999). For proc mixed, the information criteria are provided automatically. For proc reg, $\mathrm{r}^{2}$ is always provided and the "restrict" option provides a ready test for the plateau.

A major criticism of linear spline models is the abrupt change in trend. In nature, most processes are considered to change continuously from one segment to another. The rate of change is smooth. The derivatives are continuous. While this is not possible for linear splines, it is possible for higher order polynomials, beginning with quadratic functions. A quadratic spline function is smooth when the derivatives are equal at the knots.

Representation of the quadratic spline requires an additional variable: X2II $=$ XII*XII.

$$
\begin{aligned}
& \text { Model } \mathrm{Y}=\mathrm{a}+\mathrm{b} * \mathrm{X}+\mathrm{b} 2 * \mathrm{X} 2+\mathrm{bII} * \mathrm{XII}+\mathrm{b} 2 \mathrm{II} * \mathrm{X} 2 \mathrm{II} \\
& \text { If } \mathbf{X}<\text { knot then } \mathbf{Y}=\mathbf{a}+\mathbf{b} * \mathbf{X}+\mathbf{b} 2 * \mathbf{X} \mathbf{2} \\
& \text { If } \mathbf{X}>\operatorname{knot} \text { then } \mathbf{Y}=\mathbf{a}+\mathbf{b} * \mathbf{X}+\mathbf{b} 2 * \mathbf{X} 2+\mathbf{b I I} * \mathbf{X I I}+\mathbf{b} 2 \mathrm{II} * \mathbf{X} 2 \mathrm{II} \\
& \mathrm{Y}=\mathrm{a}+\mathrm{b} * \mathrm{X}+\mathrm{b} 2 * \mathrm{X} 2+\mathrm{bII} *(\mathrm{X}-\mathrm{KNOT})+\mathrm{b} 2 \mathrm{II} *(\mathrm{X}-\mathrm{KNOT}) * * 2 \\
& \mathrm{Y}=(\mathbf{a}-\mathrm{KNOT} * \mathrm{bII}+\mathrm{KNOT} * \mathrm{KNOT} * \mathrm{~b} 2 \mathrm{II})+(\mathbf{b}+\mathbf{b I I}-2 * \mathrm{KNOT} * \mathrm{~b} 2 \mathrm{II}) * \mathrm{X}+(\mathrm{b} 2+\mathrm{b} 2 \mathrm{II}) * \mathrm{X} 2
\end{aligned}
$$

This model has a single constant for continuity at the knot and a linear and quadratic coefficient for each segment. Equating the first derivatives from each segment insures a smooth function

$$
\mathrm{b}+2 * \mathrm{~b} 2 * \mathrm{X}=(\mathrm{b}+\mathrm{bII}-2 * \mathrm{KNOT} * \mathrm{~b} 2 \mathrm{II})+2 *(\mathrm{~b} 2+\mathrm{b} 2 \mathrm{II}) * \mathrm{X}
$$

and leads to a test for the smoothness restriction, $\mathrm{H}_{0}$ : $\mathrm{bII}=0$.

\section{2c.) Spline Model with Knot Unknown}

The focus of this study is to estimate the heat threshold, the knot. This problem is more difficult because the knot is a parameter of the model. Fortunately, nonlinear regression allows us to deal 
with this complication. Additional input for the regression is required. The nonlinear process is iterative and as such needs initial starting values for all parameters. Initial starting values are readily available from the known knot spline model.

Both SAS procedures, proc nlin and nlmixed, were used for this model. The nlmixed procedure (with fixed effects only-animal effects ignored) was preferred because it automatically provides the information criteria and appeared less sensitive to violations of smoothness, at least for the data in this study. The following statements for the plateau-quadratic spline are:

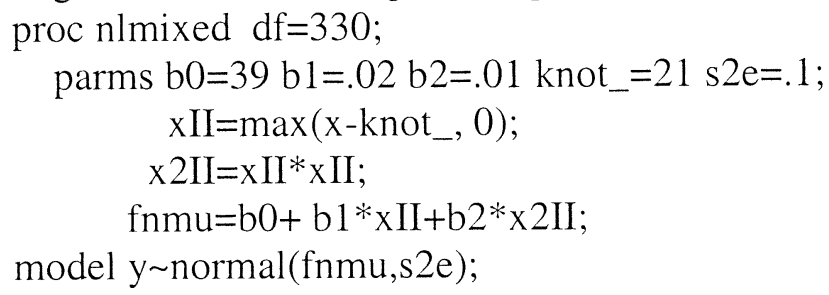

\section{Hysteresis}

Hysteresis is the retardation or delay in the effect of a thermal challenge on an animal's thermoregulatory response. The primary reason for incorporating hysteresis into the model is to recognize the time-dependence of the level of an animal's Tb for a specified thermal condition. The Tb depends on whether Ta is increasing or decreasing. For Tb, the amount of hysteresis also indicates the degree of thermoregulatory control, which is related to the animal's heat dissipation capabilities and ultimately to productive performance and survival.

A simple way to show the thermal hysteresis, i.e. the relationship between $\mathrm{Tb}$ and $\mathrm{Ta}$ as it varies with time, is to plot $\mathrm{Tb}$ against Ta. The trajectories from one point to another are then mapped to trace out the path as time passes. These graphs are known as phase plane plots and if the trajectories form ellipsoids, the process is periodic with a limit cycle that represents stable equilibrium. If there is no tilt to the ellipsoid, as in the thermoneutral stage, (Figure 6-TN), there is little correlation between $\mathrm{Tb}$ and Ta.

If the ellipsoid is tilted, as in the heat stress stage, (Figure 6-HS), there is a correlation between $\mathrm{Tb}$ and Ta. When the ellipsoid is very narrow, there is no hysteresis, no lag. If the major axis is long relative to a short minor axis, there is virtually no difference in Tb between challenge (increasing Ta) and recovery (decreasing Ta) during heat stress. As the major axis decreases and the minor axis increases, the difference between challenge and recovery becomes more variable indicating the presence of hysteresis, Figure 7 . Figure 8 shows the sinusoidal functions of Ta and $\mathrm{Tb}$ with a delay of 4 hours for the ellipsoid of lag 4 . The amount of hysteresis (lag) indicates the degree of thermoregulatory control. A lag of $4 \mathrm{~h}$ indicates less control than a lag of $1 \mathrm{~h}$. The difference between challenge and recovery is more variable for a $4 \mathrm{~h}$ lag than a $1 \mathrm{hr}$.

One way to handle hysteresis is to omit the recovery data from the analysis. The first difference can be used to classify the observations. If the first difference is less than 0 , the observation is categorized as recovery; otherwise, as challenge. Figure 9 illustrates how the pattern of the plateau-quadratic spline changes when the recovery data are omitted from the analysis. Clearly, accounting for the hysteresis improves the results. The threshold estimate is higher, closer to the theorized value, while the mse and information criteria are lower. 


\section{Nonlinear Mixed model Analysis}

There is considerable variation among the six steers, which is easily seen when the same scale is used for each plot, (Figure 10). The nonlinear mixed model procedure allows us to estimate random coefficients in addition to fixed effects. Random effects can enter the model in several ways. Inspection of Figure 10 suggests a random component to be added to the intercept for each steer and possibly a random component for the slope, bL. It is also possible to estimate the covariance between these random effects. The expected value of the two random effects is assumed to be zero.

The proc nlmixed statements for the nonlinear fixed model are given above. Proc nlmixed has additional statements when random effects are included. The two random components, steer and $\mathrm{bL}$, are specified in the random statement. They are assumed to have a normal distribution with means of zero and a covariance matrix to be estimated. The subject variable indicates new realizations of the random components occur for each animal. Additional initial parameter estimates are needed for the variance and covariance of these random effects. The following statements are for the linear-quadratic spline model:

$$
\begin{aligned}
& \text { proc nlmixed; } \\
& \text { parms b0 }=39 \mathrm{bl}=.02 \mathrm{bII}=.01 \mathrm{~b} 2 \mathrm{II}=.07 \mathrm{knot}=25 \mathrm{~s} 2 \mathrm{e}=.1 \\
& \text { s2steer }=.1 \mathrm{~s} 2 \mathrm{bL}=.05 \operatorname{cov} 12=0 \text {; } \\
& \text { bounds s2e s2s s2bL }>0 \text {; bounds } 10<\text { knot_ }_{-}<36 \text {; } \\
& \mathrm{xII}=\max (\mathrm{x}-\mathrm{knot}, 0) \text {; } \\
& \mathrm{x} 2 \mathrm{II}=\mathrm{xII} * \mathrm{xII} \\
& \mathbf{b L}=\mathbf{b} 1+\mathrm{bL} \text {; } \\
& \mathrm{fnmu}=\mathrm{b} 0+\mathrm{bL}_{-} * \mathrm{x}+\mathrm{bII} * \mathrm{xII}+\mathrm{b} 2 \mathrm{II} * \mathrm{x} 2 \mathrm{II}+\text { steer; } \\
& \text { model y } \sim \text { normal (fnmu,s2e); }
\end{aligned}
$$

\section{random steer bL normal $([0,0],[\mathrm{s} 2 \mathrm{steer}, \operatorname{cov} 12, \mathrm{~s} 2 \mathrm{bL}])$ subject=animal;}

\section{Results and Discussion}

A quadratic polynomial model for all the data gives a mse $=0.1234$ and relatively high information criteria $(\mathrm{AIC}=411.7, \mathrm{BIC}=415.9)$. Evaluation of the knot produced an unrealistic estimate of $7.3 \mathrm{C}$ for the heat stress threshold (Figure 3 ) which is much lower than the generally accepted estimates of 22-25C (Hahn, 1989).

Examples of the plateau-linear and linear-linear splines are given in Figure 4.a and 4.b. The lower mse, AIC and BIC and higher $\mathrm{r}^{2}$ indicate the linear-linear spline is the preferred model. Figures $4 \mathrm{c}$ and $4 \mathrm{~d}$ are examples of quadratic-quadratic and linear-quadratic splines. For the quadratic-quadratic model, the coefficient of bII is nonsignificant ( $p>0.05)$. The function is continuous at the estimated knot (threshold $=21.5 \mathrm{C}$ ) and there is a smooth change in trend between parabolas. On the other hand, the change in trend is discontinuous for the linearquadratic spline $(\mathrm{p}<0.05)$ leading to a model of questionable validity. The quadratic-quadratic spline is also preferred for this example because of the lower mse, AIC and BIC and higher $\mathrm{r}^{2}$.

Figures 5a-d show examples of the four spline models considered above; only this time, the knot is estimated from the data. All of these models have lower information criteria and lower estimates for the knot threshold than when the knot was specified as $21.5 \mathrm{C}$. None of the models 
satisfy the smoothness restriction. The linear-quadratic spline, with a threshold estimate of 16.89C has the best fit of all models considered thus far.

The results of the linear-quadratic spline models, both ignoring and accounting for the effects of hysteresis are given in Table 1. The data consisted of hourly means for the six steers. Proc nlmixed was used to analyze the nonlinear fixed model by omitting the random effects. The initial parameter estimates are specified in section $3 \mathrm{~b}$ above. If the effect of hysteresis is ignored, the preferred model is the plateau-quadratic spline. The information criteria are uniformly lower and the mse $=0.110$ is slightly higher. The threshold estimate is $22.67 \mathrm{C}$ with a standard error of 2.77 .

When the effect of hysteresis is managed by analyzing the challenge data, the linear-quadratic spline is preferred based on the information criteria ( $\mathrm{AIC}=70.6$ and $\mathrm{BIC}=90.4$ ). The mse is 0.085 and the threshold estimate is $26.5 \mathrm{C}$ with a standard error of 1.14 resulting in a confidence interval of $[24.31,29.71]$.

Table 2 gives the results for spline models with the knot specified as 21.5. Regardless of how hysteresis is handled, the information criteria indicate the plateau-quadratic spline is preferred. But these models pale in comparison to the splines where the knots are estimated from the data using nonlinear procedures (Table 1). Not surprisingly, the information criteria are improved (lowered) when knots are estimated from the data, regardless of whether hysteresis is ignored or managed. The specification of a $21.5 \mathrm{C}$ threshold is too high for some models and too low for others.

None of the above models pass the test for smoothness. The linear coefficient for the second segment is significant. This result casts doubt on the validity of the models and leads us to question the assumption that the rate of change is continuous. Possibly a three-segment model would be more appropriate. See Figure 11. The thermoneutral segment, TN, may be considered as a horizontal ellipse; the heat stress segment, a tilted ellipse. The middle segment could be a straight line joining the two major axes; the top of the TN axis and the bottom of the HS axis.

To include animal variation in the analysis, the Nlmixed procedure was used on the individual steer data with the random effects described in section 4 . The results for the linear-quadratic spline for the challenge data are presented in Table 3. All of the fixed parameter estimates were significant $(\mathrm{p}<0.05)$. The threshold estimate $(26.54 \mathrm{C})$ had a standard error of 0.60 . The confidence interval is $[24.89,28.21]$. However, none of the random effects were significant. The $\mathrm{mse}=0.132$ is higher than in the nonlinear fixed analysis as are the information criteria $(A I C=1035.0$ and $B I C=1033.1)$. The increase in parameters does not result in a substantial reduction in the mse.

\section{Summary}

Regressions between $\mathrm{Tb}$ and Ta provide rewarding ways to study the change between thermoneutral and heat stressing Ta. Polynomials and polynomial spline models provide estimates of heat stress thresholds. The traditional approach of using least squares to fit a polynomial (quadratic) and estimate the optimal value gave spurious results. A better approach is to use spline models. When fitting a two-segment polynomial model, the best fits are obtained 
when the second segment is a quadratic, and the first is either a plateau or linear function. Although spline models with knots given can produce reasonable fits using linear least squares, spline models with unknown knots using nonlinear regression are even better, especially if the emphasis is on estimating the threshold.

The presence of hysteresis is readily apparent from Tb-Ta plots of individuals or means over individuals. Analyses that ignore hysteresis provide poorer fits than those that recognize the presence of the effect. A plausible way to manage hysteresis when using polynomial segments is to divide the data into two categories, challenge and recovery, depending on whether the first difference is increasing or decreasing. The recovery data is then omitted from the analysis. When hysteresis was managed in this way, the optimum two-segment polynomial model for this data was the linear-quadratic spline with fixed effects. Including random effects due to steers and linear slopes to account for animal variation did not substantially improve the fit. Thus, the linear-quadratic spline with fixed effects is proposed as the best choice of the models studied. It

provides a reasonable threshold estimate with minimum standard error and optimum information criteria.

However, it must be noted that all models studied failed to pass the smoothness restriction of continuous rate of change. This suggests other models may be preferable. One possibility suggested by the data is an ellipse-linear-ellipse spline. But the most important result of this study is the need for a more explicit definition of heat stress threshold. It is important to formulate a detailed description of what comprises the change from thermoneutral to heat stress in terms of the Tb-Ta dynamics.

\section{References}

Freund, R.J. and R.C.Littell, 2000 SAS@ System for Regression, $3^{\text {rd }}$ ed SAS Institute Inc. Cary NC

Hahn, G.L. 1989, "Body temperature rhythms in farm animals." Proceedings of $11^{\text {th }}$ ISBCongress, SPB Academic Publishing, The Hage, The Netherlands. 325-337

Mader, T. L., S. M. Holt, J. B. Gaughan, G. L. Hahn, M. S. Davis, A. M. Parkhurst, and D. E. Spiers. 2001. "Heat load management for feedlot cattle". Proc. 6th Intl. Livest. Envir. Symp., Amer. Soc. Agric. Eng., St. Joseph, MI. p. 1474

SAS Institute Inc. 1999 SAS/STAT version 8, Cary NC: SAS Institute Inc

Smith, P.L. 1979, "Splines as a useful and convenient statistical tool", The American Statistician 33:57-62 
Figure 1. Pattern Obscured by Animal Variation: All Steers vs Single Steer

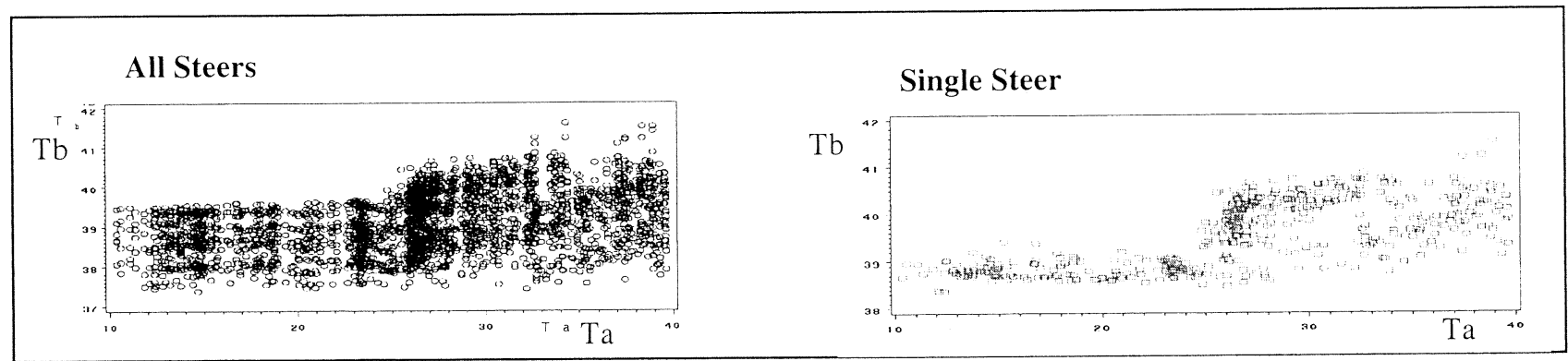

Figure 2. Sample Data with Polynomial ${ }^{\mathrm{a}}$ and Spline Models ${ }^{\text {bc }}$

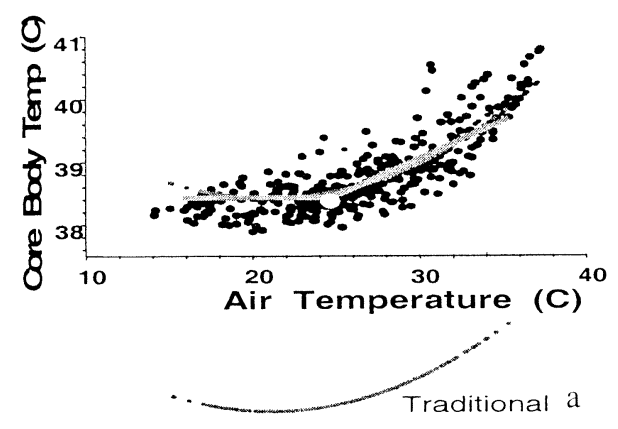

Piecewise Polynomials $\mathrm{bc}$

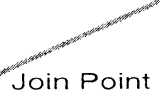

Figure 3. Quadratic Polynomial Estimate of Threshold for a Single Steer

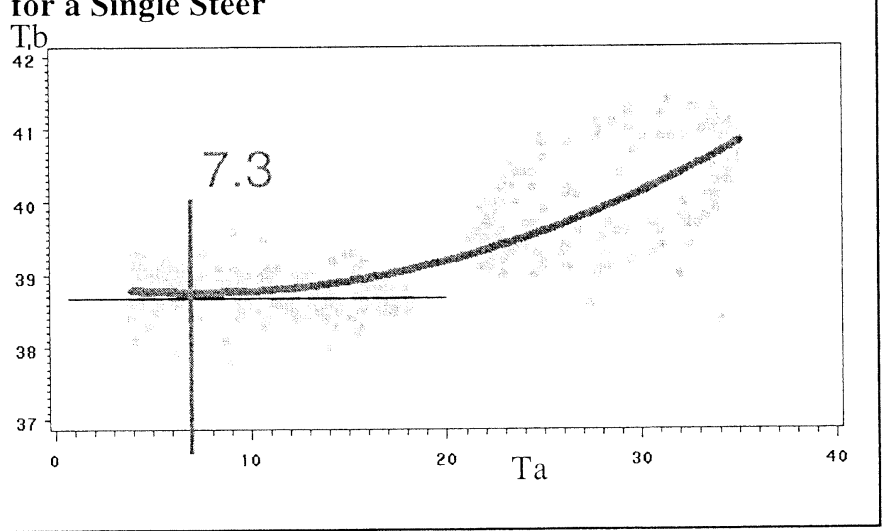


Figure 4. Examples of Spline with Knot $=21.5 \mathrm{C}$ for a Single Steer
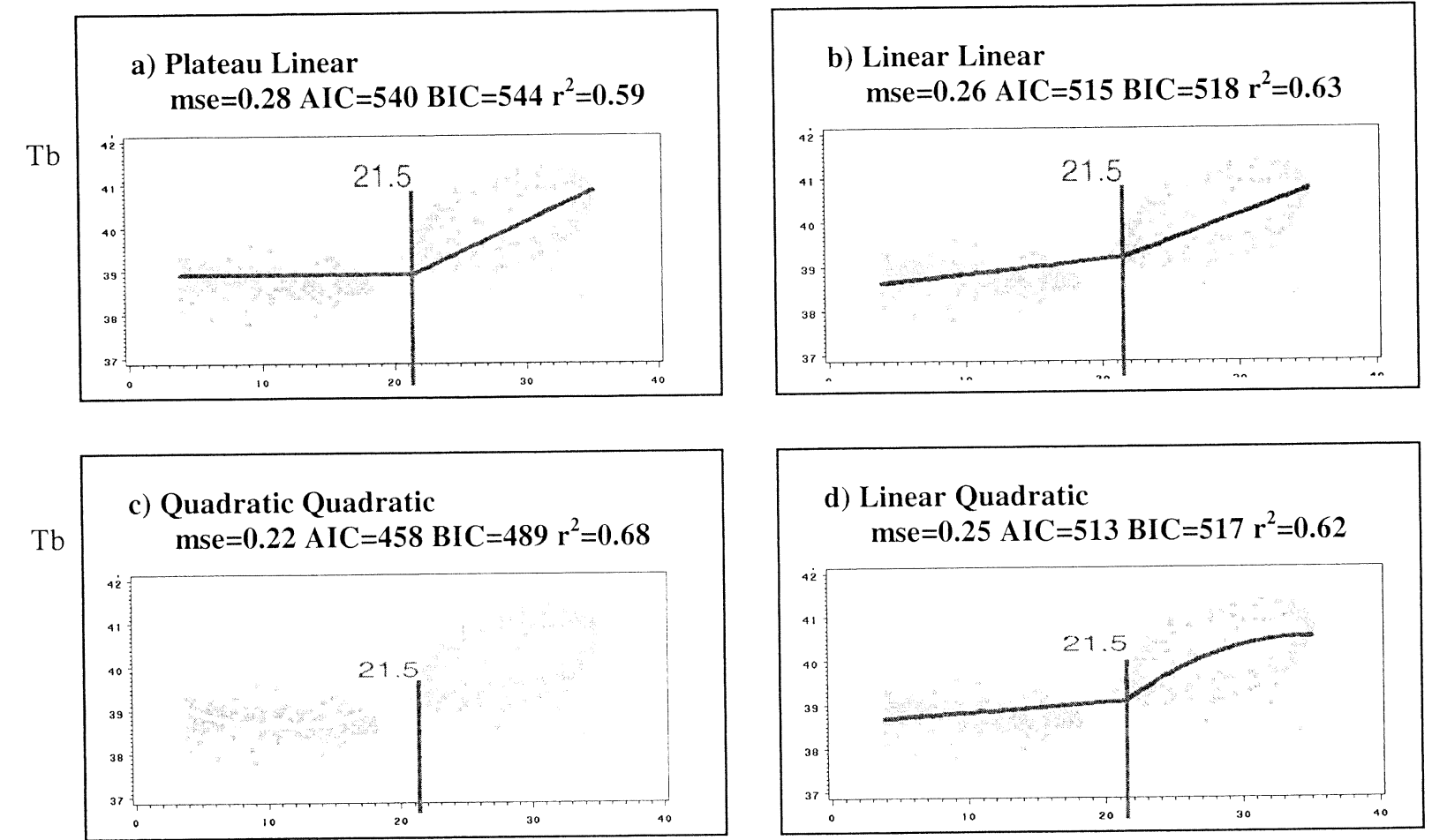

$\mathrm{Ta}$

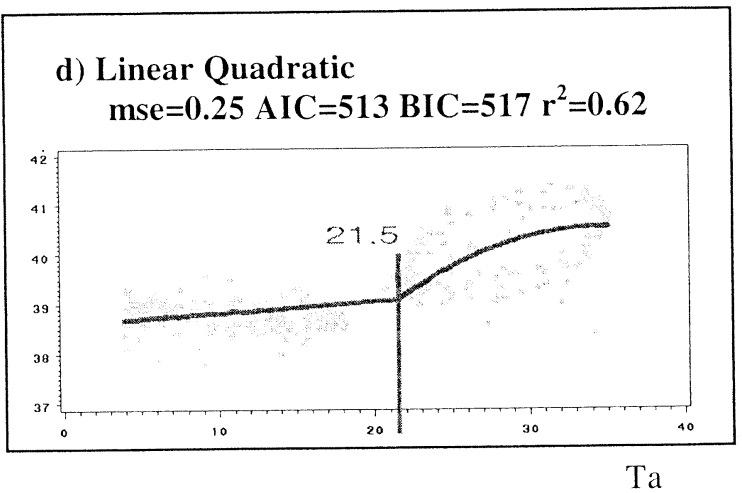

Figure 5. Example of Spline with Knot Unknown for a Single Steer
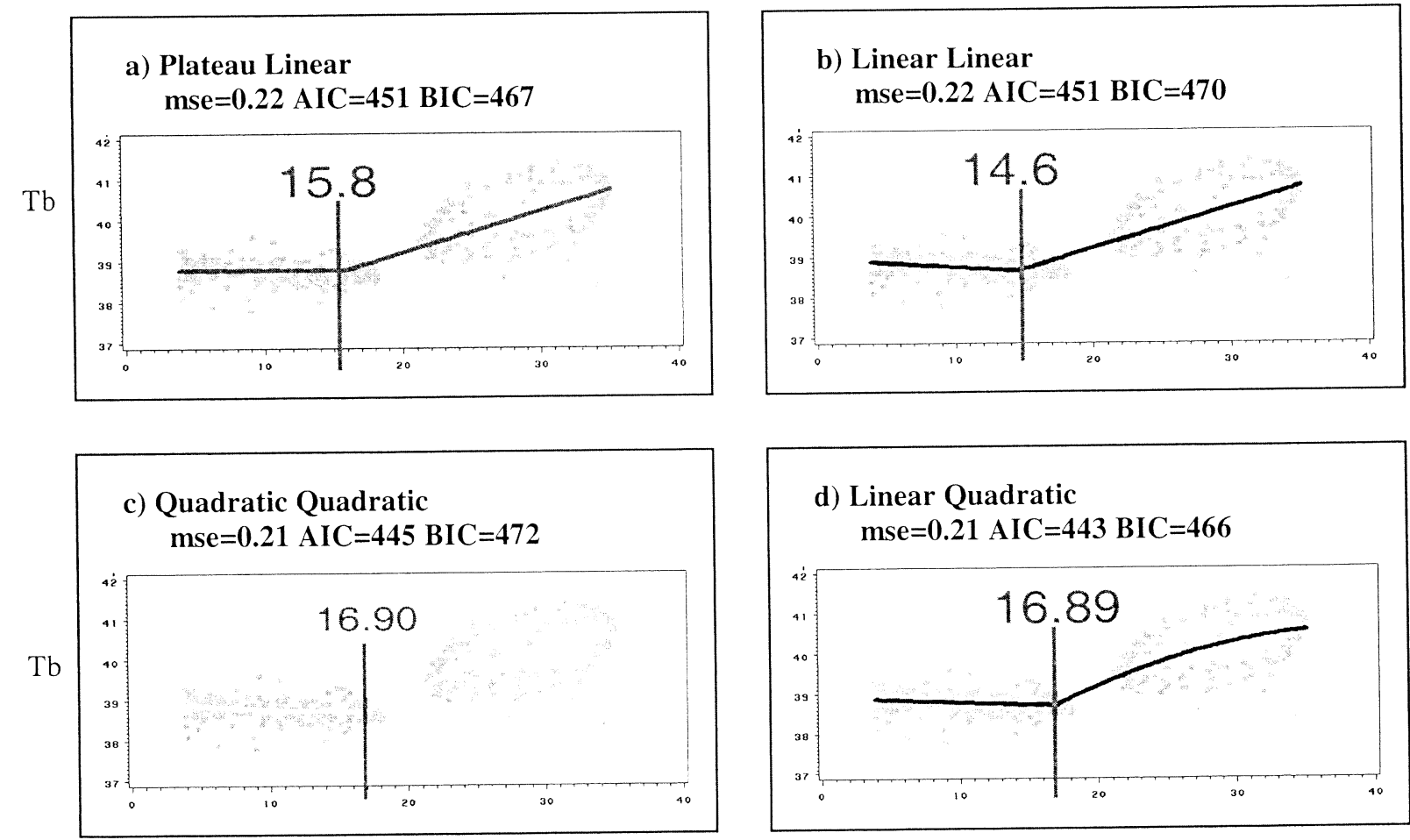

$\mathrm{Ta}$

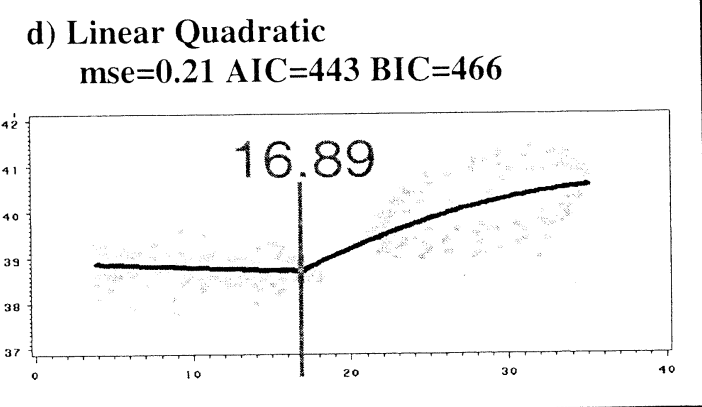


Figure 6. Difference Between Tb Challenge and Recovery for Given Level of Ta

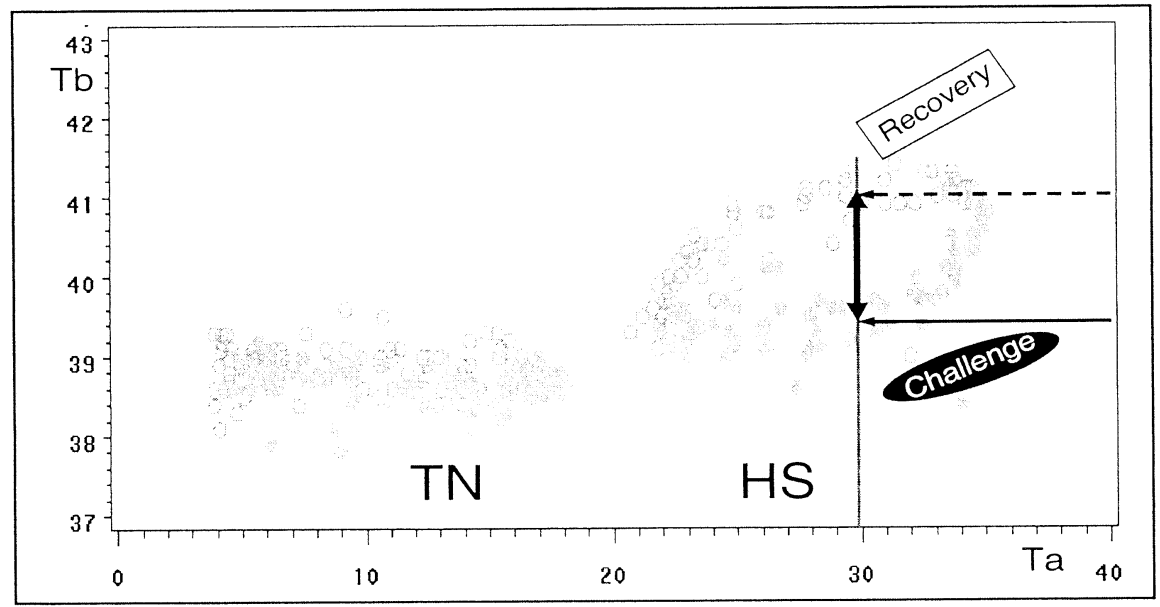

Figure 7. Change in Axes by Lag

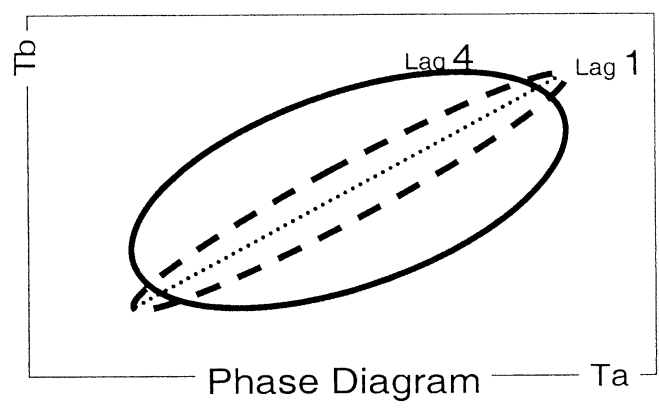

Figure 8. Effect of Ta on Tb: 4 H Delay

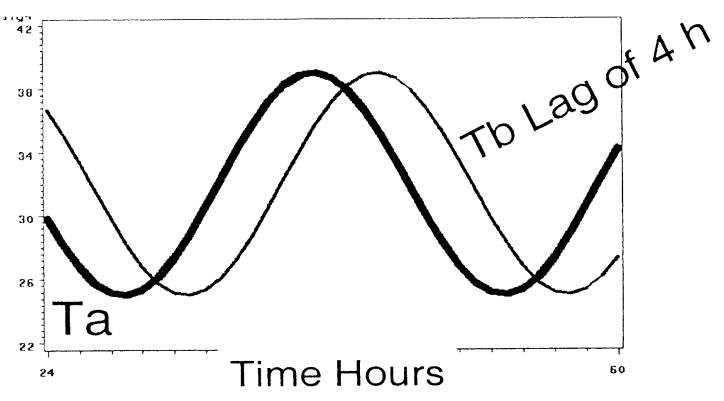

Figure 9. Threshold and Patterns Ignoring and Managing Hysteresis Plateau- Quadratic Spline Model
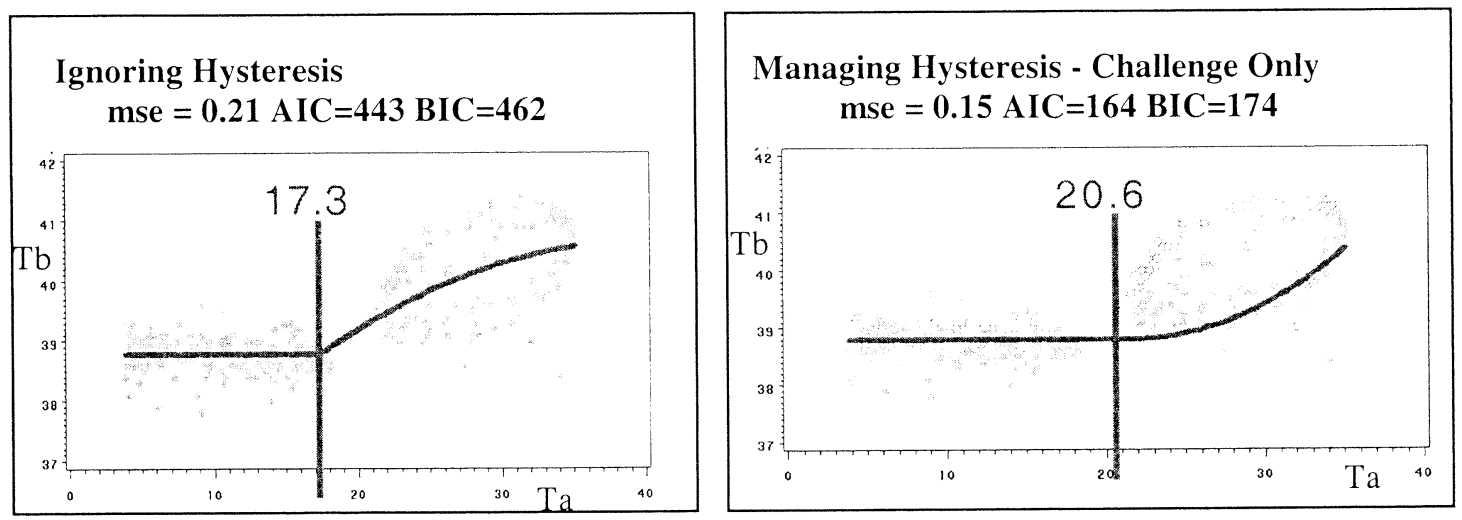
Figure 10. Animal Variation: Six Steers Same Scale

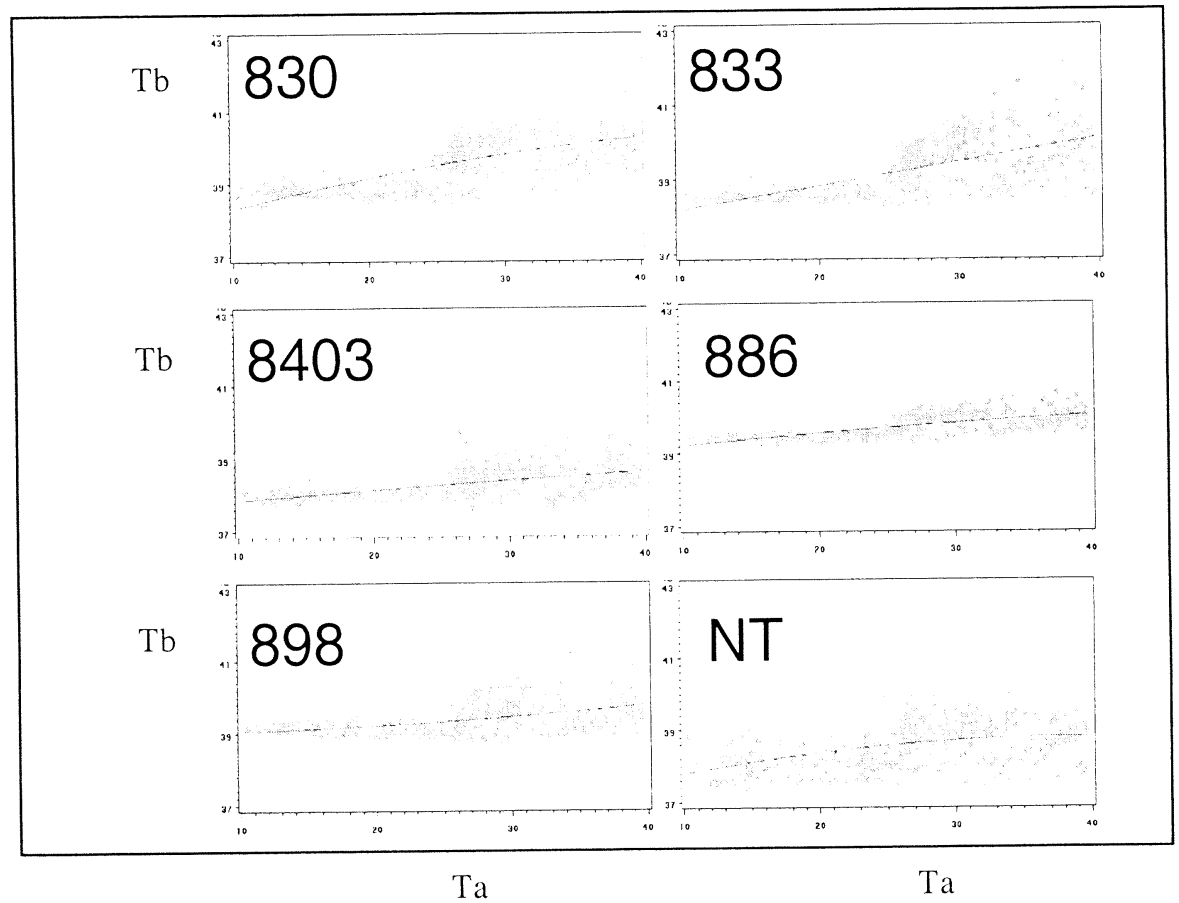

Figure 11. Possible Three-Segment Spline Model

Join End of TN and Beginning of HS with Straight Line

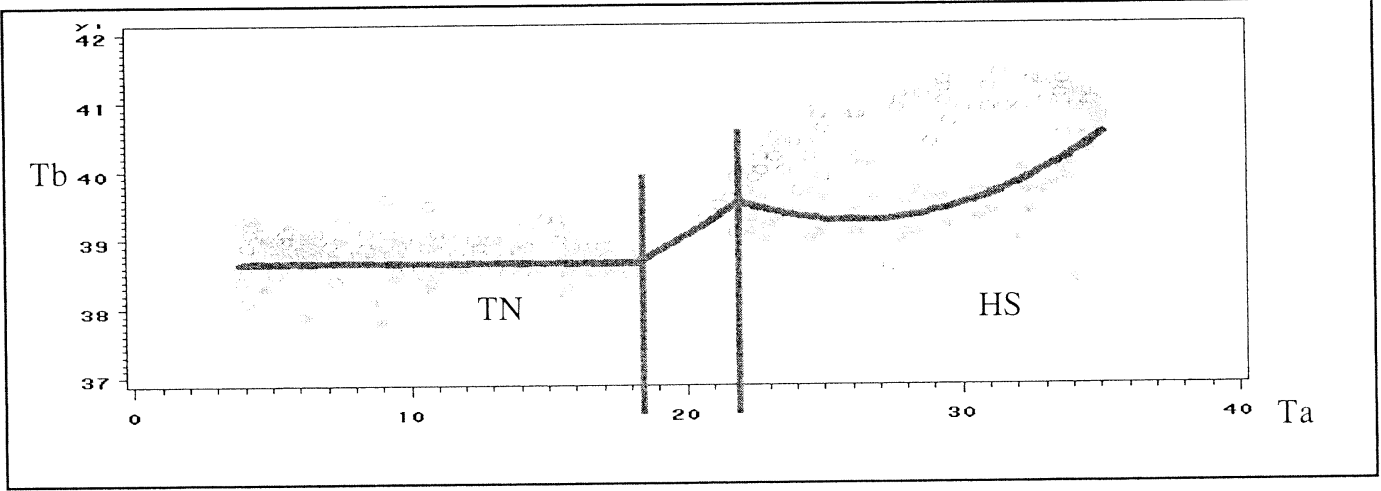


Table 1. Splines Ignoring and Managing Hysteresis Using Nonlinear Fixed Effects Model ( $\mathrm{P}=$ plateau, $\mathrm{L}=$ linear, $\mathrm{Q}=$ quadratic)

\begin{tabular}{|c|c|c|c|c|c|}
\hline $\mathrm{AIC}$ & AICC & $\mathrm{BIC}$ & mse & Model & Knot \\
\hline \multicolumn{4}{|c|}{ Ignoring } & \multicolumn{2}{|l|}{ Hysteresis: } \\
\hline 317.4 & 317.6 & 346.6 & 0.111 & $\mathrm{QKQ}$ & 22.2579 \\
\hline 315.7 & 315.9 & 340.8 & 0.111 & LKQ & 22.5459 \\
\hline 313.8 & 314 & 334.7 & 0.111 & $\mathrm{PKQ}$ & 22.6675 \\
\hline \multicolumn{3}{|c|}{ Hyster esis } & using & Challenge & Data \\
\hline 82.3 & 82.9 & 105.3 & 0.083 & QKQ & 19.599 \\
\hline 70.6 & 71.1 & 90.4 & 0.085 & LKQ & 26.5492 \\
\hline 78.4 & 78.7 & 94.8 & 0.084 & $\overline{P K Q}$ & 19.7082 \\
\hline
\end{tabular}

Table 2. Spline Models with Knot Given ( $P=$ plateau, $L=$ linear, $Q=q u a d r a t i c)$

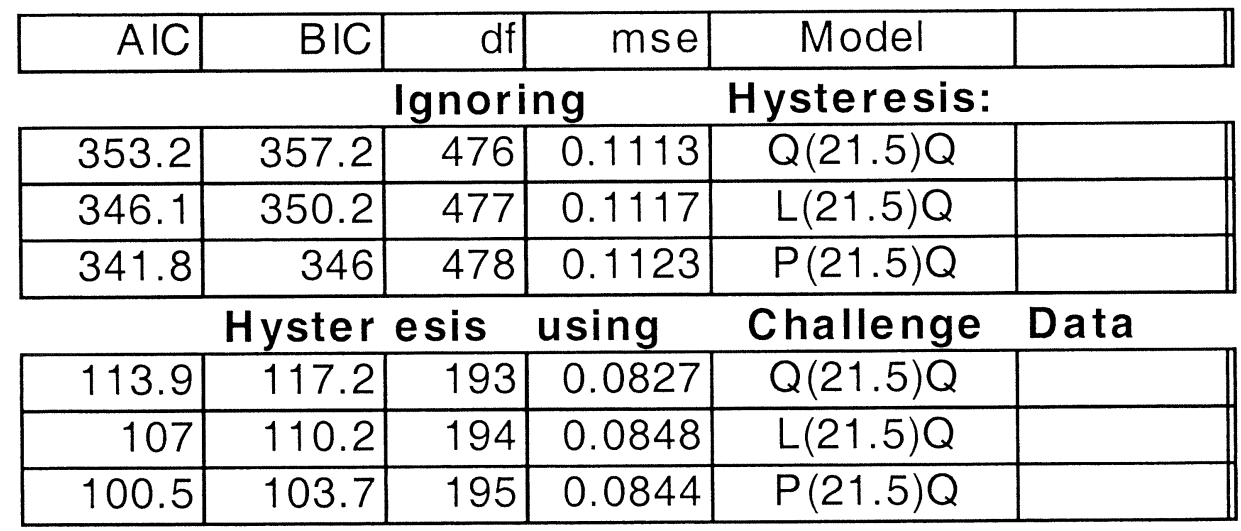

Table 3. Linear-Quadratic Spline Model NLMIXED Results for Hysteresis: Challenge $\mathrm{Y}=\mathrm{b} 0+(\mathrm{b} 1+b L) * \mathrm{X}+\mathrm{bII} * \mathrm{XII}+\mathrm{b} 2 \mathrm{II} * \mathrm{X} 2 \mathrm{II}+$ steer $+e$

\begin{tabular}{|c|c|c|c|c|c|c|c|c|}
\hline Parameter & Estimate & $\begin{array}{c}\text { Standard } \\
\text { Error }\end{array}$ & DF & t Value & $\operatorname{Pr}>|t|$ & Alph & Lower & Upper \\
\hline b0 & 38.0178 & 0.2406 & 4 & 158.04 & $<.0001$ & 0.05 & 37.3499 & 38.6857 \\
\hline b1 & 0.03472 & 0.007355 & 4 & 4.72 & 0.0092 & 0.05 & 0.01430 & 0.05514 \\
\hline bII & -0.07460 & 0.01216 & 4 & -6.13 & 0.0036 & 0.05 & -0.1084 & -0.04083 \\
\hline b2II & 0.006929 & 0.000946 & 4 & 7.32 & 0.0018 & 0.05 & 0.004303 & 0.009556 \\
\hline knot_ & 26.54 & 0.5985 & 4 & 44.36 & $<.0001$ & 0.05 & 24.88 & 28.21 \\
\hline $\mathrm{s} 2 \mathrm{e}$ & 0.1317 & 0.005435 & 4 & 24.24 & $<.0001$ & 0.05 & 0.1166 & 0.1468 \\
\hline s2steer & 0.3127 & 0.1857 & 4 & 1.68 & 0.1674 & 0.05 & -0.2028 & 0.8282 \\
\hline $\mathrm{s} 2 \mathrm{bL}$ & 0.000245 & 0.000148 & 4 & 1.66 & 0.1729 & 0.05 & -0.00017 & 0.8282 \\
\hline $\operatorname{cov} 12$ & -0.00417 & 0.004128 & 4 & -1.01 & 0.3693 & 0.05 & -0.01563 & 0.007289 \\
\hline
\end{tabular}

